\section{Disaster Research: Why, How, and When?}

Marvin L. Birnbaum, MD, $P h D$

University of Wisconsin-Madison, USA

Disaster Medicine is a new science and the development of a science requires information. As a science, we modify what we do in a given circumstance based on the validity and reliability of what we have learned through research integrated with our own experience. Such actions are tempered by the resources available or potentially available. Furthermore, it is not possible to obtain additional resources or generate change without supporting data.

Disasters are increasing in frequency, intensity, and scale. The damage resulting from an event can be assessed in terms of human, economic, and intangible costs. The costs of the damage have been skyrocketing. Obtaining accurate and reproducible information from each of these catastrophes is essential in order to mitigate these costs. Activities to mitigate the damage from future events may be directed toward elimination or modification of the hazards, decreasing the risks for actualization of the hazards (pre-event status), and/or in responses to the event from the initial responses through recovery and rehabilitation and constitute the objectives of disaster research and evaluation.

Disaster research requires the use of techniques that differ substantially from the randomized, controlled prospective, experimental studies used in traditional medical research. Qualitative research techniques (structured interviews, surveys, case-controlled studies) form the basis of the research done in the social sciences and combinations of qualitative and quantitative techniques are used in epidemiological studies. Similarly, the use of severity scores will become important. Thus, to accomplish good research in this field, we must learn new techniques and sampling strategies: ones that have high external validity, good internal validity, and high reliability. Fortunately, such techniques are easy to learn and use. The results from their use has modified our approaches to subsequent events. The design of such studies is discussed in detail as the 3rd Template in the Guidelines for Evaluation and Research in the Utstein Style. ${ }^{1}$ Future Congresses such as this should include training in these techniques.

The sooner that a study is conducted after the event (if sudden-onset), the better will be the information obtained, as the information is perishable. Ideally, such studies should be done concurrently; but this raises some ethical issues, particularly in acute-onset events. Concurrent studies have great utility in the later stages of sudden-onset disasters and in delayed-onset or chronic types of disasters. Examples of successful projects will be discussed.

The time of anecdotal reports is past: the information obtained without a structure for data collection and analysis only serves to re-iterate what we already know and generally contribute little to furthering our science. Such reports do not provide us with the information we need to create change or muster necessary resources. Disaster Medicine inter-relates with many other societal entities, and often such studies can be conducted as joint projects with members of related disciplines. The tools needed are readily at hand. The Guidelines ${ }^{1}$ will provide the structure needed for the conduct of such activities.

\section{Reference}

1. TFQCDM, Chair: Sundnes KO: Health disaster management: Guidelines for evaluation and research in the Utstein style: Executive summary. Prebosp Disast Med 1999:14;43-52.

Keywords : costs; data collection; disaster medicine; guidelines; research; science; structure; techniques; timing; training 